\title{
On General Results for All-to-All Broadcast
}

\author{
Ming-Syan Chen, Senior Member, IEEE Computer Society, Jeng-Chun Chen, and \\ Philip S. Yu, Fellow, IEEE Computer Society
}

\begin{abstract}
All-to-all broadcast refers to the process by which every node broadcasts its certain piece of information to all other nodes in the system. In this paper, we develop all-to-all broadcast schemes by dealing with two classes of schemes. A prior scheme based on generation of minimal complete sets is first described, and then a new scheme based on propagation of experts is developed. The former always completes the broadcasting in the minimal number of steps and the latter is designed to minimize the number of messages. Performance of these two classes of schemes is comparatively analyzed. The all-to-all broadcast scheme desired can be derived by combining the advantages of these two classes of schemes.
\end{abstract}

Index Terms-All-to-all broadcast, message passing, NODUP, expert propagation, communication steps.

\section{INTRODUCTION}

T NSTEAD of using a shared memory and a global clock, the 1 synchronization and communication between the processing nodes in a distributed system can be done via message passing. Because of the distributed nature of data distribution, special schemes are usually required to perform various distributed computations. One such scheme in distributed computations is broadcasting, which refers to a process of information dissemination in a distributed system where a message originating from a certain node is sent to all other nodes in the system [13], [17]. In addition to one-to-all broadcasting, all-to-all broadcasting, where every node, instead of a certain node as in one-to-all broadcasting, has a piece of information to be shared with others, is also very important in numerous applications in distributed computing [4], [12]. Applications of all-to-all broadcasting include decentralized consensus protocols [3], extrema finding, acquisition of a new global state among mobile hosts [8], and the broadcasting of various systemdependent messages [9], [12].

Note that all-to-all broadcast is the same as the gossiping problem in [7] except that a concurrent two-way transmission, such as a phone conversation, is assumed for the latter. In the context of computer networking, one-way transmission is usually adopted to represent an action of pointto-point transmission, thus leading to very different solutions from those derived for gossiping problems. Several studies have been conducted to minimize the number of message steps of one-to-all and all-to-all broadcasting schemes for various networks/distributed environments. A survey can be found in [7]. To reduce the number of messages, some broadcasting schemes and consensus protocols were proposed in [3], [11], [18] and shown to be efficient in terms of message complexity. To reduce the overhead of the scheme without compromising its efficiency, we would

- The authors are with IBM Thomas J. Watson Research Center, P.O. Box 704, Yorktown, NY 10598.

E-mail: \{mschen, jcchen, psyu\}@watson.ibm.com.

Manuscript received Aug. 24, 1994; revised May 26, 1995.

For information on obtaining reprints of this article, please send e-mail to: transactions@computer.org, and reference IEEECS Log Number D95106. naturally like to complete the broadcasting in the minimal number of steps while incurring as few messages as possible. As pointed out in [7], [14], [16], to reduce the cost of transmission, some schemes are so designed that they do not have duplicate information, i.e., every message conveys only new information to its receiver. This sort of scheme is termed "NODUP" (standing for no duplication) in [7]. In [4], a class of all-to-all broadcasting schemes was derived based on generation of minimal complete sets ${ }^{1}$ and shown to be optimal among all NODUP schemes in the sense that both the number of communication steps and the number of messages it requires are minimum under the NODUP model. An example of all-to-all broadcast among 5 nodes based on the scheme derived in [4] is shown in Fig. 1, where there are 3 steps and 12 messages incurred. The information collected thus far after each step is shown in the bracket next to each node. An arrow pointing from node $N_{i}$ to node $N_{i}$ represents that $N_{i}$ is sending what it knows thus far to $N_{i}$. For ease of exposition, we use the identification (id) of each node to denote the information that this node wants to broadcast to every other node. One message might consist of more than one id. All-to-all broadcasting is completed in the end after each node receives all the id's from all other nodes. The total number of id's sent is equal to $5+$ $10+5=20$ (i.e., Step $1+$ Step $2+$ Step 3), which, as proved in [4], is the minimum for all-to-all broadcast among 5 nodes. $^{2}$

Note that depending on the application, the real content of id can be very general, such as a number to be sorted for the global maximum, a vector describing the local state, or a yes/no vote of the commit protocol in a distributed transaction, to name a few. Thus, a message based on $\left\{a_{1}, a_{2}, \ldots\right.$, $a_{i}$ ) can actually be viewed as an application-dependent function $f\left(a_{1}, a_{2}, \ldots, a_{i}\right)$ in some applications, meaning that every message size is approximately the same and is not proportional to the number of id's involved. Such opera-

1. As defined in [4], a minimal complete set is the minimal set that has the entire information.

2. In fact, every NODUP scheme has the property of incurring the minimal number of id's sent, i.e., $p(p-1)$, in a system of $p$ nodes. 
tions are called reduction operations [6]. In view of this, the motivation of restricting our attention to NODUP schemes, which minimizes the number of id's sent, is not justified for those applications. Note that without being confined to the NODUP model one may further reduce the number of messages while still completing the all-to-all broadcast in the minimal number of steps. An example all-to-all broadcast for a system of 5 nodes is shown in Fig. 2, where same as in Fig. 1 every node can only send out one message in each step. While not being a NODUP scheme, it requires 3 steps and only 11 messages, one message fewer than that required in Fig. 1. As can be seen later, the number of messages one can reduce by relaxing the NODUP assumption can be very significant. Furthermore, this problem relaxation can lead to the development of more general solutions to all-to-all broadcast. However, despite its importance, the general problem of reducing the number of messages required for all-to-all broadcast in the minimal number of steps has not been solved. Consequently, we shall address this general problem in this paper.

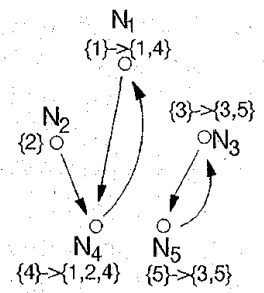

(a) Step 1

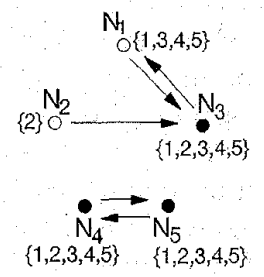

(b) Step 2

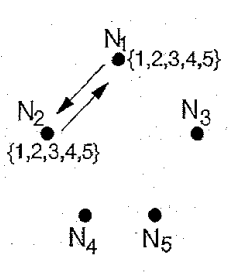

(c) Step 3
Fig. 1. A NODUP all-to-all broadcasting scheme in a system of 5 nodes ( 3 steps and 12 messages).

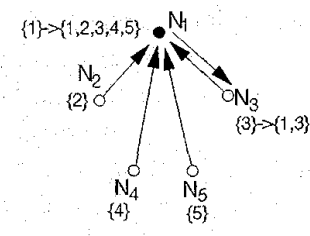

(a) Step 1

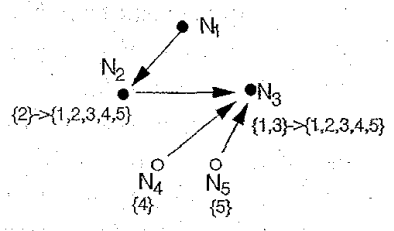

(b) Step 2

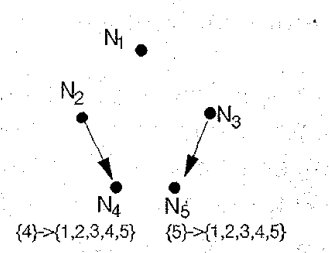

(c) Step 3

Fig. 2. An all-to-all broadcasting in a system of 5 nodes ( 3 steps and 11 messages).

To facilitate our presentation, we start with considering the case of one-port communication, which means that each node can only send out one message in one communication step. We then show our results for the case of multi-port communication, i.e., $k$-port communication, meaning that each node can simultaneously send out $k$ messages in one communication step. Also, we consider completely connected systems with synchronous communication. Such a model was employed in other related work [4] [7], [18] (A) detailed description of the model can be found in Section 2.) The problem studied in this paper can be illustrated by the examples in Figs. 1 and 2. A node having all the information from other nodes is called an expert 171. We use black nodes to denote experts, and white nodes to denote those that still have incomplete information In fact, Fig 1 corre sponds to the scheme based on generation of minimal complete sets, and Fig. 2 corresponds to the one based on propagation of experts we shall derive in this paper. To derive general results for all-to-all broadcasting, we shall deal with two classes of all-to-all broadcasting schemes that have different advantages: one is based on generation of minimal complete sets, $G_{k^{\prime}}$ and the other is based on propagation of experts, $E_{k^{\prime}}$ where $k$ is the number of messages one node can send out in one message step. Note that $G_{k}$ is a class of NODUP schemes derived in [ 4 ], and $E_{k}$ is a general scheme developed in this paper Performance of these two classes of schemes is comparatively analyzed. As will be seen later, $G_{k}$ though always leading to the minimal number of steps, may incur more messages than $E_{\text {, }}$ On the other hand, $E_{k}$, while incurring fewer messages than $G_{k}$ in many cases, may not complete the all-to-all broadcast in the minimal number of steps for certain numbers of nodes. As a result, to minimize the number of messages for all-to-all broadcasting in the minimal number of steps, one has to combine the advantages of these two classes of schemes to form the desired one.

This paper is organized as follows Preliminaries are given in Section 2. We develop general all-to-all broadcasting schemes for 1 -port and k-port communication in Sections 3 and 4 , respectively. This paper concludes with Section 5 .

\section{Preliminaries}

The model and assumptions are given in Section 2.1. Prior NODUP algorithms, $G_{1}$ and $G_{k}[4]$, are presented in Sections 2.2 and 2.3 , respectively.

\subsection{Model and Assumptions}

As stated earlier, we use the identification (id) of each node to denote the information that this node wants to broadeast to every other node. Also, the information at each node means the set of id's that node collects thus far, and the content of the message of a transmission is referred to as the information of the sender at the time of transmission the term communication step and the term message step are used interchangeably: An all to-all broadcasting is said to be completed if all nodes in the system receive all id's in the system. The system model we consider is summarized as follows.

\subsubsection{Model M}

1) The system is completely connected with synchronous communication.

2) Every message sent in the system takes one commu- 
nication step. (This assumption is supported by the techniques of virtual cut-through and wormhole routing for communication [1], [10].)

3) Reduction operations are considered, i.e., messages are approximately of the same size. As a result, general schemes are explored, not confined to NODUP (no duplication) which minimizes the total number of id's carried.

4) $k$-port communication means that each node is capable of sending $k$ messages out in one step.

5) There is no restriction on the number of messages each node can receive in one step.

Note that the provisions required for synchronization are dependent upon individual applications (such as extrema finding in a multiprocessor system, achieving a new global state among mobile hosts, crash recovery, and system initialization, to name a few). In addition, the last assumption is supported by the sufficient input buffer space and also the simplicity of the CPU operations to handle the incoming data. Explicitly, it is noted that when users write message passing code in the put/get (i.e., send/receive) model, we could usually have software bottleneck in puts rather than gets since most receives could be translated into local memory access. As a consequence, under such a put/get model which is provided by some existing message passing tools [6], fan-in could be less a restriction than fanout in message passing. This is the very reason that we are interested in the above model. While it is understood that not a single model could cover all communication paradigms, the exact expressions of message numbers we shall derive for Model $M$ are of theoretical importance and will provide reasonable upper/lower bounds for other communication models (i.e., those with different $\mathrm{I} / \mathrm{O}$ restrictions).

DEFINITION 1. An all-to-all broadcasting scheme is called optimal, if under the above model, $\mathrm{M}$, the following two conditions are satisfied.

1) It completes the broadcasting in the minimal number of steps.

2) It incurs the minimal number of messages required to complete the broadcasting in the minimal number of steps.

The objective of this paper is thus to develop effective all-to-all broadcasting schemes under model $M$.

\subsection{Description of $G_{1}$}

To facilitate the presentation of $G_{1}$, it is necessary to introduce a balanced binary partitioning tree of a positive number. A balanced binary partitioning tree of a positive number $p$ is a binary partitioning tree constructed by first labeling the root node with $p$, and then, for each node with a label $k \geq 2$, generating the left and right children of this node and labeling them with $\left\lceil\frac{k}{2}\right\rceil$ and $\left\lfloor\frac{k}{2}\right\rfloor$, respectively. Clearly, there are $n+1$ levels in the balanced binary partitioning tree of a number $p$ where $n=\left\lceil\log _{2} p\right\rceil$. For convenience, the level of the root is called level 0 . Using the balanced binary partitioning tree, the nodes in the system can be addressed as follows. For a system of $p$ nodes, obtain the balanced binary partitioning tree of $p$. Next, for every internal node, code the link to its left child with a bit " 0 " and that to its right child with a bit " 1 ". Then, determine the address of each leaf node by the coded bits in the links on the path from the root to that node, and append a bit "**" to each leaf node in level $n-1$. Finally, assign the $p$ nodes in the system with the addresses of the $p$ leaf nodes in the balanced binary partitioning tree.

Under this addressing scheme, $G_{1}$ is then executed by having each node send its message to its designated neighbor in each step. To illustrate the operations of $G_{1}$, consider a system of 10 nodes. An illustrative example for the message passing under $G_{1}$ is shown in Fig. 3 . It can be verified that the broadcasting is completed in 4 steps and the total number of messages sent is $10+10+10+4=34$. Note that for a node with an address of the form $b_{n} b_{n-1} \ldots b_{2}{ }^{*}$, such as node $010^{*}$ in Fig. $3 b$, it determines its message receiver by setting ${ }^{*}$ to 0 and inverting the appropriate bit so that each node sends out one message at a time. It can be seen that $G_{1}$ is in fact a generalization of the message exchange algorithm for hypercubes that involves swap operations among nodes in successive dimensions. Then, we have the following two propositions for $G_{1}$.

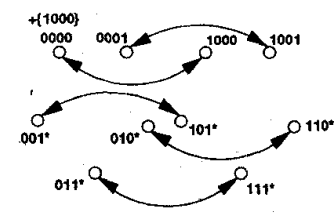

(a) Step 1: 10 messages

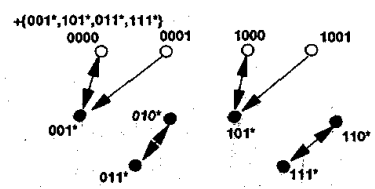

(c) Step 3: 10 messages

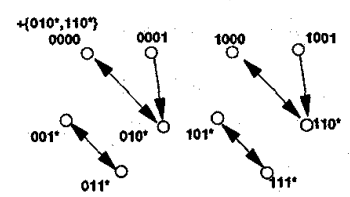

(b) Step 2: 10 messeges

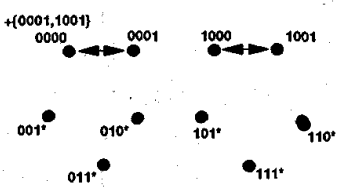

(d) Step 4: 4 messages
Fig. 3. Operations of $G_{1}$ for a system of 10 nodes.

PROPOSITION 1. For a system of $p$ nodes with one-port communication, algorithm $\mathrm{G}_{1}$ completes an all-to-all broadcasting in $\mathrm{n}$ steps by incurring $n p+p-2^{n}$ messages, where $n=$ $\left\lceil\log _{2} p\right\rceil$.

PROPOSITION 2. In a system of $p$ nodes with one-port communication, algorithm $G_{1}$ requires the minimal number of steps, $\left\lceil\log _{2} p\right\rceil$, to complete an all-to-all broadcasting.

\subsection{Description of $G_{k}$}

The results for $G_{1}$, based on the partitioning tree and the generation of minimal complete sets, can be extended to the case of $k$-port communication, meaning that each node is capable of sending $k$ messages at a time. The extension to the $k$-port communication, i.e., algorithm $G_{k}$ can be depicted in light of the generalized n-dimensional c-ary hypercube [2], where $c$ is chosen to be $k+1$. As shown in [4], all-to-all broadcasting for 2-port communication can be developed from a ternary partitioning tree in such a way that each 
internal node in level $i$ of the tree is taken as a minimal complete set generated after step $i$ and the minimal complete set associated with an internal node is partitioned into those with its child nodes in one step. Essentially, the above scheme based on generation of minimal complete sets in the corresponding partitioning tree is applicable to the case of $k$-port communication, thereby resulting in algorithm $G_{k}$. We then have the following two propositions for $G_{k}[4]$.

PROPOSITION 3 . In a system of p nodes with k-port communication, the minimal number of steps required for all-to-all broadcasting is $\left\lceil\log _{k+1} p\right\rceil$.

PROPOSITION 4. For a system of $p$ nodes with $k$-port communication, the number of messages required by algorithm $G_{k}$ for all-to-all broadcasting in $n=\left\lceil\log _{k+1} p\right\rceil$ steps is,

$$
\begin{aligned}
& N_{G}(p, k)=(d-2) n_{1} p+(d-1)\left[n_{2} p+p-(d-1)^{n_{1}} d^{n_{2}}\right] \\
& \text { where } n_{1}+n_{2}=n=\left\lceil\log _{k+1} p\right\rceil \text {, and } d \text { is the smallest } \\
& \text { positive integer such that } p \leq(d-1)^{n_{1}} d^{n_{2}} \text { and } \\
& p>(d-1)^{n_{1}+1} d^{n_{2}-1} \text {. }
\end{aligned}
$$

\section{All-To-All Broadcast For 1-PorT COMMUNICATION}

All-to-all broadcast for 1-port communication is studied in this section: As mentioned earlier, by relaxing the NODUP assumption, one can further reduce the number of messages required. We develop in Section 3.1 an all-to-all broadcasting scheme based on expert propagation, $E_{1}$. General results from combining $E_{1}$ and $G_{1}$ are given in Section 3.2 .

\subsection{Description of $E_{1}$}

The basic idea of expert propagation is to first form some expert(s) and then propagate the expert information to all other nodes. As described below, $E_{i}$. depending on the number of nodes, is devised to form either an expert in the first step (such as in Case 1), or three experts in the first two steps (such as in Case 2), and then complete the all-to-all broadcasting by recursively doubling.

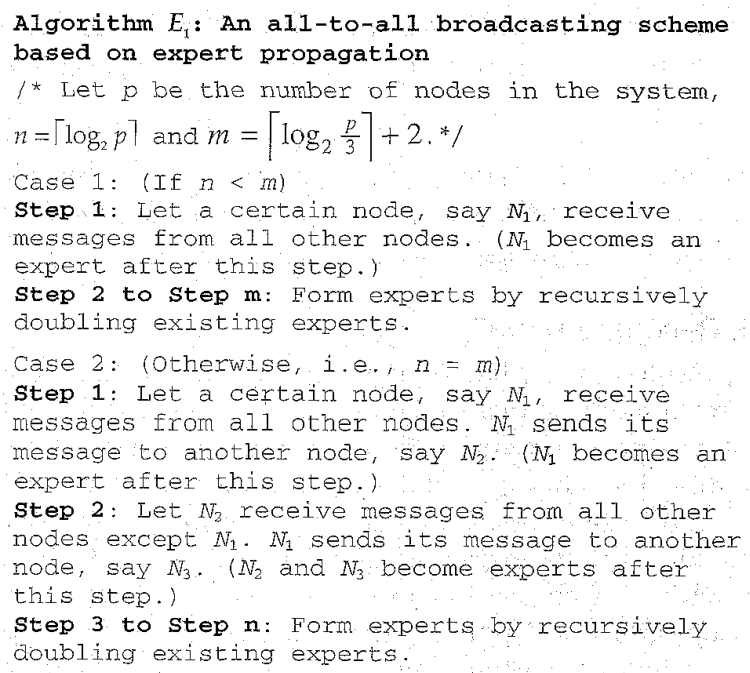

The scenario for Case is straightforward

$$
\left\lceil\log _{2} 7\right\rceil=3<\left\lceil\log _{2} \frac{7}{3}\right\rceil+2=4
$$

An example for Case 2 of $E_{1}$ is given in Fig. 4 where

$$
\left\lceil\log _{2} 10\right\rceil=\left\lceil\log _{2} \frac{10}{3}\right\rceil+2=4 \text {. }
$$

It can be observed from $E_{\text {that }}$ a node can become an expert by either receiving all ids from other nodes (such as $N_{1}$ in Case 1 and $N_{1}$ and $N_{2}$ in Case 2) or being promoted by other experts. The former method is called id collection and the latter is called expert propagation. Then, performance of $E_{1}$ can be analyzed by Lemma 1 below

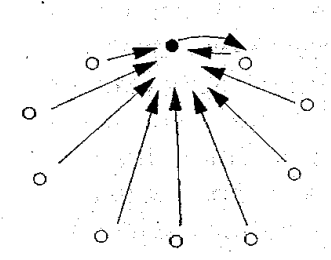

(a) Step 1

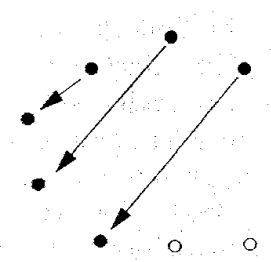

(c) Step 3

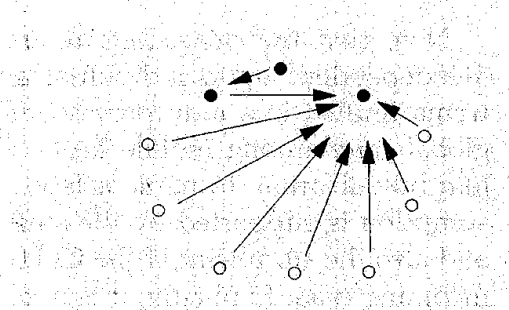

(b) Step 2
Fig. 4. An all-to-all broadcasting in a system of 10 nodes ( 4 steps and 26 messages):

LEMMA 1. For a system of p nodes with one-port communication, the number of messages reguired by algorithm $E_{1}$ for all-toall broadcasting in $m=\left[\log _{2} \frac{p}{3}\right]+2$ steps is

1) If $\left\lceil\log _{2} p\right\rceil<m$ then

$$
N_{E}(p, 1)=2 p-2
$$

2) Otherwise (i.e., $\left\lceil\log _{2} p\right\rceil=m$ ),

$$
N_{E}(p, 1)=3 p-4
$$

PROOF. Case 1 follows from the fact that there are $p-1$ mes sages incurred in Step 1 and also a total of $p-1$ mes sages incurred from Step 2 to Step $m$. Next, consider Case 2 For Case 2 the maximal number of experts obtainable after the first 2 steps is 3 . The number of steps required for all-to-all broadcasting in a system of $p$ nodes is thus $\left[\log _{2} \frac{2}{3}\right]+2$. There are 2 nodes becoming experts by $1 d$ collection, and $p-2$ nodes becoming experts by expert propagation. The total number of message is therefore equal to $2(p-1)+p$ $2=3 p-4$.

It can be seen from the all-to-all broadcasting in Fig. 4 that there are 10 messages in Step 1,9 messages in step 2,3 messages in Step 3 , and 4 thessages in Step 4 . We hence 
have $10+9+3+4=26$ messages and $\left\lceil\log _{2} \frac{10}{3}\right\rceil+2=4$ steps, agreeing with Case 2 of Lemma 1. Performance of $G_{1}$ and $E_{1}$ for various number of nodes in the system is shown in Fig. 5. In Fig. 5, triangles and squares are used to denote the points where the numbers of message steps are increased by one for $G_{1}$ and $E_{1}$, respectively. It is important to see that the dependency on two formulas for $E_{1}$, as described in Lemma 1, results in the discontinuity of the curve for the number of messages required by $E_{1}$. It can be seen that the number of message steps associated with $G_{1}$ and Case 2 of $E_{1}$ are minimal whereas those with Case 1 of $E_{1}$ are not. Lemma 2 below states the optimality of $E_{1}$ in terms of the number of messages incurred.

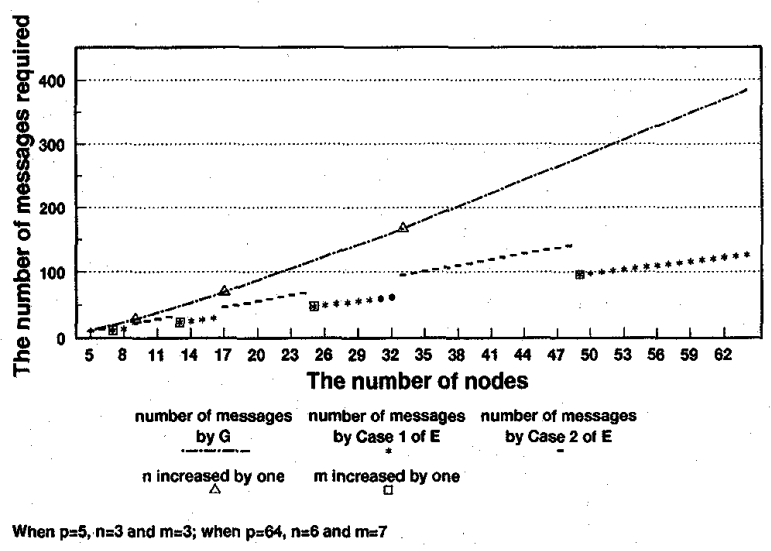

Fig. 5. The number of messages for $G_{1}$ and $E_{1}$.

LEMMA 2. For a system of $p$ nodes with one-port communication, $E_{1}$ incurs the minimal number of messages required for allto-all broadcasting in $m=\left\lceil\log _{2} \frac{p}{3}\right\rceil+2$ steps.

PROOF. Since for any scheme it always requires $p-1$ messages to form the very first expert, we know that every all-to-all broadcasting for $p$ nodes requires at least 2(p-1) messages. The optimality of Case 1 then follows. Next, we shall prove the optimality of Case 2, where $m=n=\left\lceil\log _{2} p\right\rceil$. From the fact that one-to-all broadcasting takes $n$ steps, it follows that each node has to send out one message in the first step, meaning that there are $p$ messages in the first step. Next, suppose there is no expert formed after the first step. Then, from the same reasoning we shall have $p$ messages incurred in the second step (so as to complete the broadcasting in the minimal number of steps). Since at most three experts could be formed in the first two steps, we get at least $p-3$ messages in the remaining $n-2$ steps, amounting to at least $3 p-3$ messages in total, which are more than that are required by Case 2 of Lemma 1. From the foregoing, it follows that one expert, say $N_{1}$, has to be formed after Step 1 to achieve the minimal number of messages for Case 2. This in turn implies that there are $2^{n-1}-1$ nodes which will become experts by expert propagation ini- tiated by $N_{1}$ from Step 2 to Step $n$. (This requires $2^{n-1}-$ 1 messages.) However, to make the other $r=p-1-\left(2^{n-1}-\right.$ 1) nodes become experts, each of these $2^{n-1}-1$ nodes has to send one message to any of those $r$ nodes. (This also requires $2^{n-1}-1$ messages.) From

$$
\begin{gathered}
n=\left\lceil\log _{2} p\right\rceil=\left\lceil\log _{2} \frac{p}{3}\right\rceil+2, \\
\text { we have } r=p-2^{n-1} \leq 2^{n-2} \text {. (Since } p-2^{n-1}>2^{n-2} \text { implies } \\
\left\lceil\log _{2} \frac{p}{3}\right\rceil+2 \geq\left\lceil\log _{2} \frac{4 p}{3}\right\rceil>n,
\end{gathered}
$$

a contradiction.) Clearly, the minimal number of messages to complete all-to-all broadcasting among $r$ nodes is $2 r-2$, meaning that we need at least $2\left(p-2^{n-1}\right)-2=2 p-2^{n}-2$ within these $r$ nodes. Consequently, the minimal number of total messages required is $p+2^{n-1}-1+2^{n-1}-1+2 p-2^{n}-2=3 p-4$, agreeing with the formula in Case 2 of Lemma 1.

\subsection{General Results From Combining $G_{1}$ and $E_{1}$}

It can be seen that $G_{1}$, though always leading to the minimal number of steps, may incur more messages than $E_{1}$. On the other hand, $E_{1}$, while incurring the minimal number of messages, may not complete the all-to-all broadcasting in the minimal number of steps for certain numbers of nodes (as stated in Case 1 of Lemma 1 when $\left\lceil\log _{2} p\right\rceil<\left\lceil\log _{2} \frac{p}{3}\right\rceil+2$ ). As a result, to minimize the number of messages for all-toall broadcasting in the minimal number of steps, one has to combine the advantages of these two classes of schemes to form the desired one. Formally, from Proposition 1 and Lemma 1, we have the following theorem.

THEOREM 1. For a system of $p$ nodes with one-port communication, the minimal number of messages required for all-to-all broadcasting in $n=\left\lceil\log _{2} p\right\rceil$ steps is

$$
M(p, 1)= \begin{cases}3 p-4, & \text { if }\left\lceil\log _{2} \frac{p}{3}\right\rceil+2=n, \\ n p+p-2^{n}, & \text { otherwise }\end{cases}
$$

\section{All-To-All Broadcast for K-Port COMMUNICATION}

In this section, we develop general results for all-to-all broadcasting schemes with k-port communication, without being restricted to NODUP schemes. Algorithm $E_{k^{\prime}}$ based on propagation of experts, is devised in Section 4.1, and general results from combining $G_{k}$ and $E_{k}$ and some remarks are given in Section 4.2 .

\subsection{Description of $E_{k}$}

We devise in this subsection an all-to-all broadcasting scheme based on expert propagation for $k$-port communication, $E_{k^{\prime}}$ to minimize the number of messages for the number of steps required. Basically, $E_{k}$ will try to form a set of $k$ experts, referred to as set $A$, in the first step. Same as the reasoning of making $N_{2}$ an expert by id collection in Case 2 of $E_{1}, E_{k}$ also makes a set of up to $k$ nodes, referred to as set $B$, become experts by id collection in Step 2 so as to complete the all-to-all broadcasting in the minimal number 
of steps. Note that the cardinality of set B is dependent on the values of $p$ and $k$, and will be determined by Lemma 3 later.

Algorithm $E_{k}$ : An all-to-a 11 broadcasting scheme based on expert propagation.

${ }^{*}$ Let $p$ be the number of nodes in the system.

$$
m=\left\lceil\log _{k+1} \frac{p}{k(k+2)}\right\rceil+2 * \text {; }
$$

step 1: Let $k$ certain nodes, say $N_{i} I \leq i \leq k$, form a set $A$, and receive messages from all other nodes. Nodes in set A exchange messages among them, and also send a message to their counter part in another set of up toknodes (set $B$ ), respectively. (Nodes in set A become experts after this step.)

Step 2: Let nodes in set B receive messages from all other nodes except those in set $A$. Nodes in set $A$ send their messages to another $k^{2}$ nodes. (Nodes in set $B$ and these $k^{2}$ nodes become experts after this step.)

Step 3 to step m: Form experts by recursively multiplying (i.e.; by ktimes) existing experts.

An example for algorithm $E_{2}$ is given in Fig. 6 where for clarity big arrows are used to denote group transmission. It can be seen that an all-to-all broadcasting in a system of 22 nodes for 2-port communication requires 3 steps and a total of 100 messages. Similarly to $E_{1}$, a node under $E_{k}$ can become an expert by either receiving all ids from other nodes (such as nodes in sets $\mathrm{A}$ and $\mathrm{B}$ ) or being promoted by other experts (such as nodes other than those in sets $A$ and B). Clearly, the more nodes becoming experts by expert propagation, the fewer messages are required. Performance of $E_{k}$ can be analyzed by Lemma 3 below.

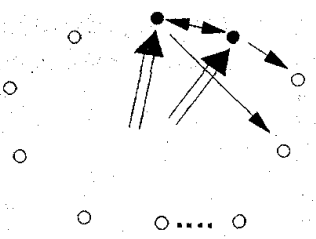

(a) Step 1

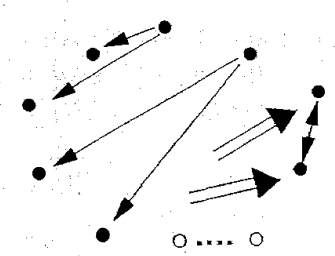

(b) Step 2

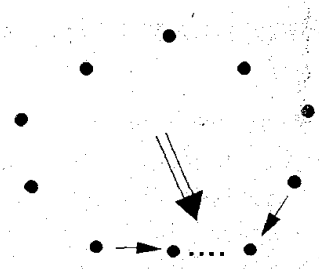

(c) Step 3

Fig. 6. E2 in a system of 22 nodes for 2-port communication (3 steps and $44+42+14=100$ messages).

LEMMA 3. For a. system of $p$ nodes with k-port communication, the number of messages required by algorithm $E_{k}$ for all-toall broadcasting in $m=\left[\log _{k+1} \frac{p}{k(k+2)}\right]+2$ steps is

$$
\text { 1) If } \begin{aligned}
\log _{k+1} \frac{p}{k} \leq m-1 \text { then } \\
N_{\bar{E}}(p, k)=b_{1}(p-1)+p-b_{1}
\end{aligned}
$$

where $b_{1}$ is the smallest positive integer such that

$$
\log _{k+1} \frac{p}{b_{1}} \leq m-1
$$

2) Otherwise,

$N_{E}(p, k)=\left(b_{2}-k-k^{2}\right)(p-k-1)+k p+p-b_{2}+k^{2}$

where $b_{2}$ is the smallest positive integer such that

$$
\log _{k+1} \frac{p}{b_{2}} \leq m-2
$$

PROOF. Case 1 corresponds to the situation that nodes only become experts via id collection in the first step (ive set $B$ in algorithm $E_{k}$ is an empty set) $b$, is the number of experts after Step 1 Each of those $b$ nodes receives $p-1$ messages, and each of the other $p-b$, nodes receives one message. We thus have $N_{E}(p, k)=b_{1}(p-1)$ $+p-b_{1}$ messages.

Case 2 corresponds to the situation that nodes can become experts via id collection in the first two steps. $b$, is the number of experts after the first two step. It can be obtained that set $A$ has $k$ nodes and set $B$ has $b-k$ $-k^{2}$ nodes. Nodes in sets $\mathrm{A}$ and $\mathrm{B}$ become experts by id collection. Note that nodes in set $A$ receive $k(p-1)$ messages in Step 1, and those in set B receive $k$ mes sages in Step 1 and $\left(b_{2}-k-k^{2}\right)(p-k-1)$ messages in Step 2 . The other $p-b_{2}+k^{2}$ nodes receive one message each (i.e, becoming experts by expert propagation) We then obtain that $N_{E}(p, k)=\left(b_{2}-k-k^{2}\right)(p-k-1)+$ $k p+p-b_{2}+k^{2}$, proving this lemma.

It can be seen from the all-to-all broadcasting in Fig. 6 that there are 44 messages in Step 1,42 messages in Step 2, and 14 messages in Step 3 . We have $44+42+14=100$ mes sages, agreeing with what we obtain from Case 2 of Lemma 3 , where $p=22, k=2, b_{2}=8$ and thus $N_{E}(22,2)=100$. It can be verified that Lemma 1 is a special case of Lemma 3 when $k=1, b_{1}=1$ and $b_{2}=3$. Performance of $G_{2}$ and $E_{2}$ for various number of nodes in the system is shown in Fig. 7 . Again, in Fig. 7, triangles and squares are used to denote the points where the numbers of message steps are increased by one for $G_{2}$ and $E_{2}$, respectively. It is interesting to see from Fig. 7 that in addition to the dependency on two cases in Lemma 3 , different values of $b_{1}$ and $b_{2}$ also result in the discontinuity of the curve for the number of messages required by $E_{2}$.

\subsection{General Results From Combining $G_{k}$ and $E_{k}$}

$E_{k}$, though incurring fewer messages than $G_{k}$ in many cases, may not complete the all-to-all broadcast in the minimal number of steps. Specifically, the number of steps required by $E_{k^{\prime}}$. i.e., $m=\left[\log _{k+1} \frac{p}{k(k+2)}\right]+2$, could be greater than the minimal, i.e, $n=\left\lceil\log _{k+1} p\right\rceil$, which can be achieved by $G_{k}$ Also, note that the general problem of determining the minimal number of messages required to complete the all to-all broadcast with $k$-port communication in certain number of message steps is not yet completely solved. To minimize the number of messages for all-to all broadcasting in the minimal number of steps for $k$ port communication, one has to combine the advantages of $G_{k}$ and $E_{k}$ to form the desired one. Proposition 4 and Lemma 3 lead to the following lemma 
LEMMA 4. For a system of $p$ nodes with $k$-port communication, the minimal number of messages required by $G_{k}$ and $E_{k}$ for all-to-all broadcasting in $n=\left\lceil\log _{k+1} p\right\rceil$ steps is

$$
M(p, k)= \begin{cases}N_{E}(p, k), & \text { if }\left[\log _{k+1} \frac{p}{k(k+2)}\right]+2=n, \\ N_{G}(p, k), & \text { otherwise }\end{cases}
$$

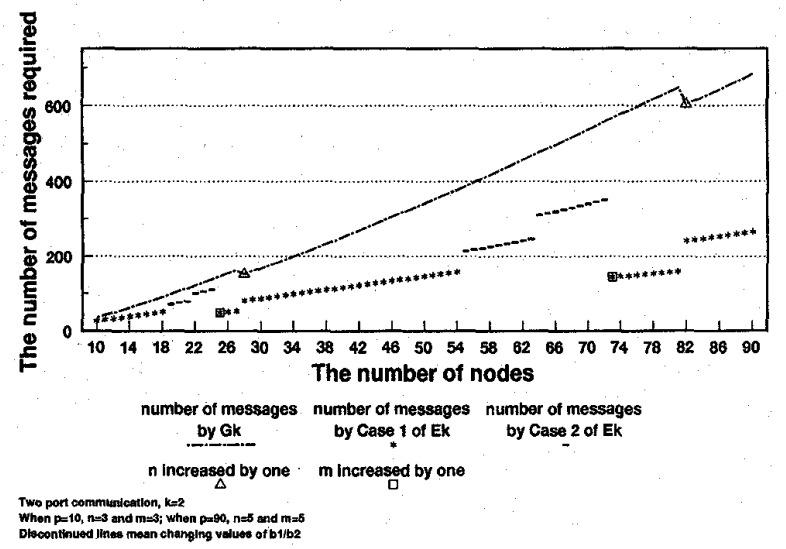

Fig. 7. The number of messages for $G_{2}$ and $E_{2}$.

A solid curve corresponding to the numbers $M(p, 2)$, for $10 \leq p \leq 90$, is shown in Fig. 8. Note that the solid curve in Fig. 8 is composed of not only the lines associated with $G_{2}$ and Case 2 of $E_{2^{\prime}}$ but also those with Case 1 of $E_{2}$. This is a result of the fact that due to the capability of multiport communication, Case 1 of $E_{2}$, though only making experts by id collection in the first step, can still complete the broadcasting in the minimal number of steps for certain numbers of system nodes.

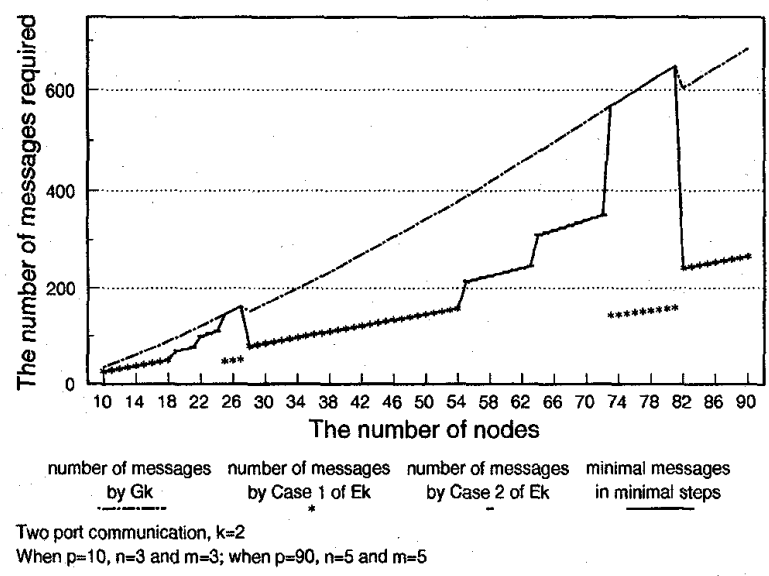

Fig. 8. The minimal number of messages from combining $G_{2}$ and $E_{2}$.

Note that same as in [4] we do not exclude either the possibility of two-way transmission between two nodes, or the capability of each node to participate in both sending and receiving messages in one communication step, thus distinguishing our work in this paper from the one in [15]. In addition, the schemes proposed in this paper are developed under the assumption that the system is completely connected and every message takes one communication step. While their variations could provide fair performance for some topologies, these schemes are not designed for all system interconnections. Certainly, when the put/get model mentioned in Section 2 is not available and the capability of input ports becomes the constraint, one would have to resort to such results as flow control [5]. It is believed that different communication paradigms will warrant different model assumptions which in turn lead to very different solutions from one toianother.

\section{Conclusion}

In this paper, we developed the all-to-all broadcasting schemes by dealing with two classes of schemes: a prior one based on generation of minimal complete sets and the new one based on propagation of experts. The all-to-all broadcasting schemes for the case of one-port communication were first developed from combining $G_{1}$ and $E_{1}$. Then, we presented our results for the case of $k$-port communication and devised all-to-all broadcasting schemes based on $G_{k}$ and $E_{k}$. To reduce the number of messages required for allto-all broadcasting in the minimal number of steps, various formulas, which can be applied to cases corresponding to different relationships between $p$ and $k$, are derived.

\section{ACKNOWLEDGMENTS}

The authors are grateful for anonymous referees and C.-T. Ho at IBM Almaden for their helpful comments on improving this paper.

\section{REFERENCES}

[1] W.C. Athas and C.L. Seitz, "Multicomputers: Message-Passing Concurrent Computers," IEEE Computer, vol. 21, pp. 9-24, Aug. 1988.

[2] L. Bhuyan and D.P. Agrawal, "Generalized Hypercube and Hyperbus Structures for a Computer Network," IEEE Transactions on Computers, vol. 33, no. 4, pp. 323-333, Apr. 1984.

[3] M.-S. Chen, P.S. Yu, and K.-L. Wu, "Decentralized Consensus Protocols With Multi-Port Communication," Proc. 13th Int'l Conf. Distributed Computing Systems, pp. 356-365, May 1993.

[4] M.-S. Chen, P.S. Yu, and K.-L. Wu, "Optimal NODUP All-to-All Broadcast Schemes in Distributed Systems," IEEE Transactions on Parallel and Distributed Systems, vol. 5, no. 12, pp. 1,275-1,285, Dec. 1994.

[5] P.B. Danzig, "Flow Control for Limited Buffer Multicast," IEEE Transactions on Software Engineering, vol. 20, no. 1, pp. 1-12, Jan. 1994.

[6] Message Passing Interface Forum, MPI: A Message Passing Interface Standard, May 1994.

[7] S.M. Hedetniemi, S.T. Hedetniemi, and A. Liestman, "A Survey of Broadcasting and Gossiping in Communication Networks," Networks, vol. 18, pp. 319-351, 1988.

[8] T. Imielinski and B.R. Badrinath, "Data Management for Mobile Computing," ACM SIGMOD Record, vol. 22, no. 1, pp. 34-39, Mar. 1993.

[9] S.L. Johnsson and C.T. Ho, "Optimum Broadcasting and Personalized Communication in Hypercubes," IEEE Transactions. on Computers, vol. 38, no. 9, pp. 1,249-1,268, Sept. 1989.

[10] P. Kermani and L. Kleinrock, "Virtual Cut-Through: A New Computer Communication Switching Technique," Computer Networks, vol. 3, pp. 267 286, 1979. 
[11] T.V. Lakshman and A.K. Agrawala, "Efficient Decentralized Consensus Protocols," IEEE Transactions on Software Engineering, vol. 12, no. 5, pp. 600-607, May 1986.

[12] S. Lee and K.G. Shin, "Interleaved All-to-All Reliable Broadcast on Meshes and Hypercube," IEEE Transactions on Parallel and Distributed Systems, vol. 5 , no. 5, pp. 449-458, May 1994.

[13] P. Ramanathan and K.G. Shin, "Reliable Broadcast in Hypercube Multicomputers, IEEE Transactions on Computers, vol. 37, no. 12, pp. 1,654-1,657, Dec. 1988

[14] A. Seres, "Quick Gossiping Without Duplicate Transmissions," Graphs and Combinatorics, vol. 2, pp. 363-383, 1986

[15] K.N. Venkataraman, G. Cybenko, and D.W. Krumme, "Simultaneous Broadcasting in Multiprocessor Networks," Proc. Int'l Conf. Parallel Processing, pp. 555 558, 1986.

[16] D.B. West, "Gossiping Without Duplicate Transmissions," SIAM J. Alg. Disc. Meth., vol. 3, pp. 418-419, 1982.

[17] C.-B. Yang, R.C.T. Lee, and W.-T. Chen, "Parallel Graph Algorithms Based upon Broadcasting Communications," IEEE Transactions on Computers, vol. 39, no. 12, pp. 1,468-1,472, Dec. 1990.

[18] S.-M. Yuan and A.K. Agrawala, "A Class of Optimal Decentralized Commit Protocols," Proc. 8th Int'l Conf. Distributed Computing Systems, pp. 234-241, 1988

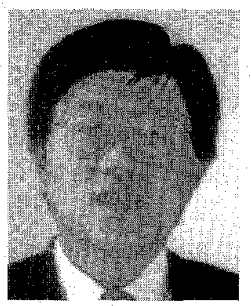

Ming-Syan Chen ( $M$ ' $88, S M$ ' 93 ) received the BS degree in electrical engineering from $\mathrm{Na}-$ tional Taiwan University, Taipei, Taiwan, Republic of China, in 1982 and the MS and PhD degrees in computer, information and control engineering from The University of Michigan, Ann Arbor, in 1985 and 1988 , respectively. He has been a research staff member at IBM Thomas J. Watson Research Center, Yorktown Heights, N.Y., since 1988 and is currently involved in projects related to multimedia.systems and database mining. From 1985 to 1988 , he was a member of the Real-Time Computing Laboratory at. The University of Michigan, Ann Arbor, where he conducted research on hypercube routing and processor allocation. His research interests include multimedia technologies, digital libraries, database systems, data mining, and combinatorial theory. Dr. Chen is a guest editor for IEEE Transactions on Knowledge and Data Engineering on a special issue for database mining in 1996. In addition to serving as a program committee member for several conferences on his research areas, he is also a conference tutorial lecturer on parallel query processing. He is an inventor of many international patents in the areas of interactive video playout, video senver design, database mining, and multiprocessor networks. He received the Outstanding Innovation Award from IBM in 1994 for his contribution to parallel transaction design and implementation for a major database product and numerous awards for his inventions and patent applications. Dr. Chen is a member of the Association for Computing Machinery.

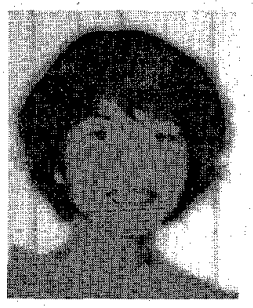

Jeng-Chun Chen received the BS degree in electrical engineering from National Taiwan University, Taipei, Taiwan, Republic of China, in 1985 and the MS degree in electrical engineering and computer science from The University of Michigan, Ann Arbor, in 1986. She has been with the IBM Thomas J. Watson Research Center, Yorktown Heights, N.Y., since 1989 and has been involved in projects on multimedia programming, image technologies, microkernel operating systems, and performance analysis of $\mathrm{PC}$ architecture. Currently, she is designing and implementing videoconferencing on personal computers. From 1985 to 1988 , she was with the Mental Health Research Institute at The University of Michigan, Ann Arbor, and conducted research on image processing and signal processing. She received the Research Division Award from IBM in 1995 for her contribution to the design and implementation of video $\mathrm{CD}$. Her current research interests include videoconferencing, digital video compression systems, and multimedia technologies.

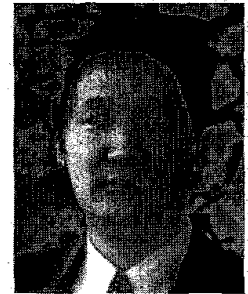

Philip S. Yu (S 76, M78, SM $\left.87, F^{\prime} 93\right)$ received the $B S$ degree in electrical engineering from National Taiwan University, Taipei, Taiwan, Repubilic of China in 1972 , the MS and PhD degrees in electrical engineering from Stanford University in 1976 and 1978 respectively, and the MBA degree from New York University in 1982. Since 1978 he has been with the IBM Thomas $J$ Watson Research Center, Yorktown Heights, N.Y. Currently he is manager of the Architecture Analysis and Design Group. His current research interests include database systems, data mining multimedia systems, transaction and query processing parallel and distributed systems, disk arrays, computer architecture, performance: modeling, and workload analysis. He has published more than 190 papers in refereed journals and conferences and over 130 research reports and 90 invention disclosures. He holds or has applied for 31 U.S, patents. Dr. Yu is a member of the ACM. He is an editor of IEEE Transactions on Knowledge and Data Engineering In addition to serving as program committee members on various conferences, he has served as the program chair of the Second International Workshop on Research Issues on Data Engineering: Transaction and Query Processing and as the program co-chair of the 11 th International Conference on Data Engineering. He has received several IBM and external honors, including Best Paper Award, IBM Outstanding Innovation Awards, Outstanding Technical Achievement Award, Research Division Award, and 15 Invention Achievement Awards. 